\title{
Everywhere regularity of certain nonlinear diffusion systems
}

\author{
Maxim Trokhimtchouk
}

Received: 11 November 2008 / Revised: 15 May 2009 / Accepted: 10 July 2009 /

Published online: 22 August 2009

(c) The Author(s) 2009. This article is published with open access at Springerlink.com

\begin{abstract}
In this paper I discuss nonlinear parabolic systems that are generalizations of scalar diffusion equations. More precisely, I consider systems of the form

$$
\mathbf{u}_{t}-\Delta[\nabla \Phi(\mathbf{u})]=0,
$$

where $\Phi(z)$ is a strictly convex function. I show that when $\Phi$ is a function only of the norm of $\mathbf{u}$, then bounded weak solutions of these parabolic systems are everywhere Hölder continuous and thus everywhere smooth. I also show that the method used to prove this result can be easily adopted to simplify the proof of the result due to Wiegner (Math Ann 292(4):711-727, 1992) on everywhere regularity of bounded weak solutions of strongly coupled parabolic systems.
\end{abstract}

Mathematics Subject Classification (2000) $\quad 35 \mathrm{~K} 40 \cdot 35 \mathrm{~K} 55 \cdot 35 \mathrm{~B} 65 \cdot 35 \mathrm{~B} 35 \cdot 35 \mathrm{D} 10$

\section{Introduction}

The theory of regularity of nonlinear scalar elliptic and parabolic equations is by now classical. It goes back to the ground breaking work on equations in divergence form by De Giorgi, Moser and Nash in the late fifties. Since then, Hölder estimates for general nonlinear elliptic and parabolic equations were derived by Krylov and Safonov. However, it became clear quite early on, after discovery of counter examples, that nonlinear elliptic and parabolic systems do not, in general, possess everywhere regularity. Instead, only partial regularity results are available [8].

Despite the lack of everywhere Hölder continuity of weak solutions of general elliptic and parabolic systems, there are several nontrivial examples that do possess everywhere regularity due to their special structure. One of the earliest such examples of a fully nonlinear elliptic system whose weak solutions are everywhere Hölder continuous is due to Uhlenbeck [12]. She considered elliptic systems of the form

M. Trokhimtchouk ( $\square)$

University of California, Berkeley, 970 Evans Hall \#3840, Berkeley, CA 94720-3840, USA

e-mail: trokhim@math.berkeley.edu 


$$
-\left(g\left(|\nabla \mathbf{u}|^{2}\right) u_{x_{\alpha}}^{i}\right)_{x_{\alpha}}=0 \text { for } i \in\{1 \ldots N\},
$$

with some additional ellipticity and grows conditions on $g$. Uhlenbeck's proof of Hölder continuity of weak solutions of these systems relies crucially on the existence of an auxiliary function which is subharmonic. This auxiliary function (entropy) is tied to $\left|\nabla^{2} \mathbf{u}\right|^{2}$ by an inequality, and this allows local control on the second derivatives of $\mathbf{u}$.

The parabolic examples of fully nonlinear systems possessing everywhere regularity followed somewhat later. DiBenedetto and Friedman first showed continuity [4] and then Hölder continuity [5] for the gradient of solution to the parabolic equation

$$
\mathbf{u}_{t}-\nabla\left(|\nabla \mathbf{u}|^{p-2} \nabla \mathbf{u}\right)=0
$$

where $\max \{1,2 N /(N+2)\}<p<\infty$. Wiegner in [13] provided another example of parabolic system with everywhere regularity. He showed everywhere Hölder continuity of weak solutions of strongly coupled systems

$$
u_{t}^{i}-\left(a_{\alpha \beta} u_{x_{\beta}}^{i}+c_{\alpha \beta}^{i} H_{x_{\beta}}\right)_{x_{\alpha}}=0
$$

with $H:=H(\mathbf{u})$ a strictly convex function of $\mathbf{u}$ and strict ellipticity conditions on the coefficients. His work has been followed by several others, including Dung [6], on strongly coupled system, and Bae and Choe [1] on a parabolic analog of the example of Uhlenbeck. Unlike Uhlenbeck's work, however, none of the proofs of everywhere regularity for aforementioned parabolic examples rely explicitly on the existence of entropy.

In this paper I provide new examples of parabolic systems whose weak solutions are everywhere Hölder continuous. These systems are a type of nonlinear diffusion systems, and are of the form

$$
\mathbf{u}_{t}-\Delta(\nabla \Phi(\mathbf{u}))=0
$$

where $\Phi(z)$ is a strictly convex function. These systems are interesting since they generalize scalar nonlinear diffusion equations of the form

$$
u_{t}-\Delta(\gamma(u))=0
$$

where $\gamma$ is strictly increasing. I will show that if $\Phi(z)$ depends only on the norm of $z$, then, together with some smoothness and grows conditions on $\Phi$, weak solutions of diffusion systems (2) are everywhere Hölder continuous. I will do this by exhibiting an entropy, and showing that existence of entropy together with properties of general parabolic systems suffices to prove everywhere regularity. In the conclusion, I will show that regularity of bounded weak solutions to the strongly coupled systems (1) can also be obtained with the help of an entropy.

Let me introduce notation that I will use throughout this paper. I consider parabolic space to consist of space and time with space being of $n$ dimensions and time of one dimension. In this paper I will deal with cylindrical domains for simplicity. By elliptic domain $\Omega \subset \mathbb{R}_{x}^{n} \mathrm{I}$ will mean space domain. Cylindrical parabolic domain $\Omega \times(0, T) \subset \mathbb{R}_{x}^{n} \times \mathbb{R}_{t}$, where $T>0$, will be denoted by $\Omega_{T}$. The time interval of the cylindrical domain $\Omega_{T}$, that is the interval $(0, T)$ in the case of $\Omega_{T}=\Omega \times(0, T)$, will be denoted by $I\left(\Omega_{T}\right)$. By $\mathrm{B}_{r}(x)$ I will mean an $n$ dimensional ball as a subset of $\mathbb{R}_{x}^{n}$ with center at $x$ and of radius $r . Q(x, t, r)$ will denote the cylinder

$$
Q(x, t, r):=\mathrm{B}_{r}(x) \times\left(t-r^{2}, t\right) .
$$

I will write $\mathrm{B}_{r}$ or $\mathrm{B}$ instead of $\mathrm{B}_{r}(x)$ when $x$ or $r$ are clear from the context. Similarly, sometimes I will write $Q_{r}$ or $Q$ instead of $Q(x, t, r)$. For a Lebesgue measurable set $S$ 
by $|S|$ I will mean Lebesgue measure of $S$. Finally $V\left(\Omega_{T} ; \mathbb{R}^{n}\right)$ will denote the closure of $C^{1}\left(\Omega_{T} ; \mathbb{R}^{n}\right)$ functions under the norm

$$
\|v\|_{V\left(\Omega_{T}\right)}^{2}=\sup _{t \in I\left(\Omega_{T}\right)} \int_{\Omega}|v(x, t)|^{2} \mathrm{~d} x+\iint_{\Omega_{T}}|\nabla v(x, t)|^{2} \mathrm{~d} x \mathrm{~d} t .
$$

\section{Partial regularity of nonlinear systems}

In this section we recall partial regularity results for weak solutions of quasi-linear parabolic systems of the form

$$
u_{t}^{i}-\left(A_{i j}^{\alpha \beta}(x, \mathbf{u}) u_{x_{\beta}}^{j}\right)_{x_{\alpha}}=0 \quad \forall i \in\{1 \ldots N\},
$$

where coefficients $A_{i j}^{\alpha \beta}$ satisfy the following condition of strong ellipticity:

$$
A_{i j}^{\alpha \beta} \xi_{\alpha}^{i} \xi_{\beta}^{j} \geq \lambda|\xi|^{2} \text { for all } \xi \in \mathbb{R}^{n N} \text {, for some } \lambda>0
$$

By a weak solution in this case I mean a function $\mathbf{u} \in V\left(\Omega_{T} ; \mathbb{R}^{N}\right)$ that satisfies

$$
\iint_{\Omega_{T}}-u^{i} v_{t}^{i}+A_{i j}^{\alpha \beta} u_{x_{\beta}}^{j} v_{x_{\alpha}}^{i} \mathrm{~d} x \mathrm{~d} t=0, \quad \text { for all } \mathbf{v} \in \mathrm{H}_{0}^{1}\left(\Omega_{T} ; \mathbb{R}^{N}\right) .
$$

It is well known that weak solutions of systems of this type possess partial regularity under some appropriate continuity conditions on $A_{i j}^{\alpha \beta}$. The important result in this area is the following local regularity result due to Giaquinta and Struwe [10].

Theorem 1 (Local regularity condition) Suppose coefficients $A_{i j}^{\alpha \beta}$ satisfy condition (4), are continuous and bounded. Also suppose $\mathbf{u} \in V\left(\Omega_{T}\right)$ is a weak solution of (3). Then, if for some $\left(x_{0}, t_{0}\right) \in \Omega_{T}$

$$
\liminf _{R \rightarrow 0} \frac{1}{R^{n}} \iint_{Q\left(x_{0}, t_{0}, R\right)}|\nabla \mathbf{u}|^{2} \mathrm{~d} x \mathrm{~d} t=0,
$$

then $\mathbf{u}$ is Hölder continuous in the neighborhood of $\left(x_{0}, t_{0}\right)$.

Condition (5) is the basis for the proofs of everywhere regularity that we will discuss in the rest of this paper. We will, however, need one more result due to Giaquinta and Struwe [10].

Lemma 1 ( $\mathrm{L}^{p}$ estimate) Let $\mathbf{u}$ be a weak solution of the system (3). Then there exists an exponent $p>2$ such that $|\nabla \mathbf{u}| \in \mathrm{L}_{\text {loc }}^{p}\left(\Omega_{T}\right)$; moreover for all $Q_{R} \subset Q_{4 R} \subset \Omega_{T}$ we have

$$
\left(\int_{Q_{R}}|\nabla \mathbf{u}|^{p} \mathrm{~d} x \mathrm{~d} t\right)^{1 / p} \leq C\left(\int_{Q_{4 R}}|\nabla \mathbf{u}|^{2} \mathrm{~d} x \mathrm{~d} t\right)^{1 / 2} .
$$

Please refer also to the work of Duzaar and Mingione [7], which provides a characterization of regular points for the spacial gradient and for more general non-linear parabolic systems. 


\section{Generalized diffusion equations}

In this section I discuss the type of parabolic systems I will refer to as diffusion system. Let $\Phi: \mathbb{R}^{N} \rightarrow \mathbb{R}$ be a strictly convex, twice continuously differentiable function with

$$
\lambda|\xi|^{2} \leq \Phi_{z_{i} z_{j}} \xi^{i} \xi^{j} \leq \Lambda|\xi|^{2} .
$$

Then we say that $\mathbf{u}$ is a weak solution of

$$
\mathbf{u}_{t}-\Delta[\nabla \Phi(\mathbf{u})]=0
$$

if $\mathbf{u} \in V\left(\Omega_{T} ; \mathbb{R}^{N}\right)$ and for all $\mathbf{w} \in \mathrm{H}_{0}^{1}\left(\Omega_{T} ; \mathbb{R}^{N}\right)$

$$
\iint_{\Omega_{T}}-u^{i} w_{t}^{i}+\left(\Phi_{z_{i}}(\mathbf{u})\right)_{x_{\alpha}} w_{x_{\alpha}}^{i} \mathrm{~d} x \mathrm{~d} t=0 .
$$

This is a standard quasi-linear elliptic system of the type in (3), since we can rewrite it as

$$
u_{t}^{i}+\left(\Phi_{z_{i} z_{j}}(\mathbf{u}) u_{x_{\alpha}}^{j}\right)_{x_{\alpha}}=0 \text { for all } i \in\{1 \ldots N\} .
$$

If in addition $\mathbf{u}$ is bounded I say $\mathbf{u}$ is bounded weak solution.

As I have mentioned before this equation is a generalization of scalar nonlinear diffusion equation. It has several nice properties. First, its flow is a contraction in $\mathrm{H}^{-1}(\Omega)$ and its solutions are unique. Second, weak solutions of (8) are in $\mathrm{H}_{l o c}^{1}\left(0, T ; \mathrm{L}_{l o c}^{2}(\Omega)\right)$, that is their weak derivatives in time are in $\mathrm{L}_{l o c}^{2}\left(\Omega_{T}\right)$. Furthermore, if the solution is bounded, the gradient in $x$ is actually in $\mathrm{L}_{\text {loc }}^{4}\left(\Omega_{T}\right)$.

First I show that flow is a contraction in $\mathrm{H}^{-1}(\Omega)$.

Theorem 2 (Uniqueness) Let $u_{0}, u_{1} \in V\left(\Omega_{T} ; \mathbb{R}^{N}\right)$ be two weak solutions of (8) with the same boundary conditions, that is $u_{0}(\cdot, t) \equiv u_{1}(\cdot, t)$ on $\partial \Omega$ for almost all $t \in[0, T]$. Denote by $i: \mathrm{L}^{2}(\Omega) \rightarrow \mathrm{H}^{-1}(\Omega)$ the natural embedding of square integrable functions in $\mathrm{H}^{-1}$ defined by

$$
i(f)(\phi):=\int_{\Omega} f \phi \mathrm{d} x .
$$

Then we have for $T \geq t_{1} \geq t_{0} \geq 0$

$$
\left\|i\left(u_{0}\left(t_{1}\right)-u_{1}\left(t_{1}\right)\right)\right\|_{\mathrm{H}^{-1}(\Omega)} \leq e^{\lambda\left(t_{1}-t_{0}\right)}\left\|i\left(u_{0}\left(t_{0}\right)-u_{1}\left(t_{0}\right)\right)\right\|_{\mathrm{H}^{-1}(\Omega)},
$$

where $\lambda$ as in (7).

Proof Let us denote by $f_{h}$ the Steklov average of $f$ defined as

$$
f_{h}(x, t):=\frac{1}{h} \int_{t}^{t+h} f(x, s) \mathrm{d} s .
$$

Also for simplicity we will write $\mathbf{v}_{k}$ for $\nabla \Phi\left(\mathbf{u}_{k}\right)$ with $k=0,1$. It is not very hard to show that $\left(\mathbf{u}_{k}\right)_{h}$ and $\left(\mathbf{v}_{k}\right)_{h}$ weakly satisfy

$$
\left(\left(\mathbf{u}_{k}\right)_{h}\right)_{t}-\Delta\left(\left(\mathbf{v}_{k}\right)_{h}\right)=0 .
$$

Let us denote the solution of

$$
\Delta w=f, \quad w \equiv 0 \text { on } \Omega
$$


by $\Delta^{-1} f$. Then the $\mathrm{H}^{-1}(\Omega)$ norm of $i(f)$ is given by

$$
\|i(f)\|_{\mathrm{H}^{-1}(\Omega)}^{2}=\int_{\Omega}\left|\nabla\left(\Delta^{-1} f\right)\right|^{2} \mathrm{~d} x .
$$

For simplicity for an $f \in \mathrm{L}^{2}(\Omega)$ let us write $\|f\|_{\mathrm{H}^{-1}}$ instead of $\|i(f)\|_{\mathrm{H}^{-1}(\Omega)}$. Now fix $h$. For $t_{0}, t_{1} \in(h, T-h)$ we compute

$$
\left.\left(e^{2 \lambda t}\left\|\left(\mathbf{u}_{1}\right)_{h}-\left(\mathbf{u}_{0}\right)_{h}\right\|_{\mathrm{H}^{-1}}^{2}\right)\right|_{t_{0}} ^{t_{1}}=I_{1}+I_{2}
$$

where

$$
I_{1}=\left.\int_{t_{0}}^{t_{1}} 2 \lambda e^{2 \lambda t}\left\|\left(\mathbf{u}_{1}\right)_{h}-\left(\mathbf{u}_{0}\right)_{h}\right\|_{\mathrm{H}^{-1}}^{2}\right|_{t} \mathrm{~d} t
$$

and

$$
I_{2}=\int_{t_{0}}^{t_{1}} \int_{\Omega} 2 e^{2 \lambda t} \nabla \Delta^{-1}\left(\left(\mathbf{u}_{1}\right)_{h}-\left(\mathbf{u}_{0}\right)_{h}\right) \cdot \nabla \Delta^{-1}\left(\left(\mathbf{u}_{1}\right)_{h}-\left(\mathbf{u}_{0}\right)_{h}\right)_{t} \mathrm{~d} x \mathrm{~d} t .
$$

Using the equation and the fact that $\mathbf{u}_{0}$ and $\mathbf{u}_{1}$ have the same trace, for $I_{2}$ we obtain

$$
I_{2}=-\int_{t_{0}}^{t_{1}} \int_{\Omega} 2 e^{2 \lambda t}\left(\left(\mathbf{u}_{1}\right)_{h}-\left(\mathbf{u}_{0}\right)_{h}\right) \cdot\left(\left(\mathbf{v}_{1}\right)_{h}-\left(\mathbf{v}_{0}\right)_{h}\right) \mathrm{d} x \mathrm{~d} t
$$

Taking the limit of both sides of the Eq. (13) as $h \rightarrow 0$ we get

$$
\left(e^{2 \lambda t} \|\left.\left(\mathbf{u}_{1}-\mathbf{u}_{0} \|_{\mathrm{H}^{-1}}^{2}\right)\right|_{t_{0}} ^{t_{1}}=I_{3}+I_{4},\right.
$$

where

$$
I_{3}=\left.\int_{t_{0}}^{t_{1}} 2 \lambda e^{2 \lambda t}\left\|\mathbf{u}_{1}-\mathbf{u}_{0}\right\|_{\mathrm{H}^{-1}}^{2}\right|_{t} \mathrm{~d} t
$$

and

$$
\begin{aligned}
I_{4} & =-\int_{t_{0}}^{t_{1}} \int_{\Omega} 2 e^{2 \lambda t}\left(\mathbf{u}_{1}-\mathbf{u}_{0}\right) \cdot\left(\mathbf{v}_{1}-\mathbf{v}_{0}\right) \mathrm{d} x \mathrm{~d} t \\
& \leq-\int_{t_{0}}^{t_{1}} \int_{\Omega} 2 \lambda e^{2 \lambda t}\left|\mathbf{u}_{1}-\mathbf{u}_{0}\right|^{2} \mathrm{~d} x \mathrm{~d} t \\
& \leq-\left.\int_{t_{0}}^{t_{1}} 2 \lambda e^{2 \lambda t}\left\|\mathbf{u}_{1}-\mathbf{u}_{0}\right\|_{\mathrm{H}^{-1}}^{2}\right|_{t} \mathrm{~d} t
\end{aligned}
$$

where the second line follows from strict convexity (7) of $\Phi$. Indeed,

$$
\left(\mathbf{u}_{1}-\mathbf{u}_{0}\right) \cdot\left(\mathbf{v}_{1}-\mathbf{u}_{0}\right)=\left(\mathbf{u}_{1}-\mathbf{u}_{0}\right) \cdot\left(\nabla \Phi\left(\mathbf{u}_{1}\right)-\nabla \Phi\left(\mathbf{u}_{0}\right)\right) \geq \lambda\left|\mathbf{u}_{1}-\mathbf{u}_{0}\right|^{2} .
$$


Thus we see that $I_{3}+I_{4} \leq 0$ and we establish the result for $T>t_{1} \geq t_{0}>0$. We establish the Theorem by taking the limit as $t_{0} \rightarrow 0$ and $t_{1} \rightarrow T$.

Remark 1 Observe that bound (7) implies that $\nabla \Phi$ is a Lipschitz map. In particular if we let $\mathbf{v}:=\nabla \Phi(\mathbf{u})$, then $\mathbf{v}_{x_{\alpha}}^{i}=\Phi_{z_{i} z_{j}} \mathbf{u}_{x_{\alpha}}^{j}$, and

$$
\left|\mathbf{v}_{x_{\alpha}}\right| \leq C\left|\mathbf{u}_{x_{\alpha}}\right| \text { for all } \alpha \in\{1, \ldots, n\},
$$

and hence

$$
|\nabla \mathbf{v}| \leq C|\nabla \mathbf{u}|
$$

for some constant $C>0$, because coefficients of the matrix $\nabla^{2} \Phi$ are uniformly bounded. Furthermore, estimate (7) implies that inverse of $\nabla^{2} \Phi$ is also uniformly bounded, hence we also have

$$
|\nabla \mathbf{u}| \leq C|\nabla \mathbf{v}|
$$

Let us now derive the $\mathrm{H}^{2}$ estimates for the solutions of (8). In general, quasi-linear parabolic systems do not have $\mathrm{H}^{2}$ estimates and the fact that solutions of generalized diffusion equations do have them is the consequence of the special structure that Eq. (8) possesses.

Theorem $3\left(\mathrm{H}^{2}\right.$ estimates) Let $u \in V\left(\Omega_{T} ; \mathbb{R}^{N}\right)$ be a weak solution of (8). Then $u_{t} \in$ $\mathrm{L}_{\text {loc }}^{2}\left(\Omega_{T}\right)$ and for $Q(x, t, r) \subsetneq Q(x, t, R) \subset \Omega_{T}$ we have the following estimates

$$
\begin{aligned}
\iint_{Q(x, t, r)}\left|\mathbf{u}_{t}\right|^{2} \mathrm{~d} x \mathrm{~d} t & \leq \frac{C}{(R-r)^{2}} \iint_{Q(x, t, R)}|\nabla \mathbf{u}|^{2} \mathrm{~d} x \mathrm{~d} t, \\
\iint_{Q(x, t, r)}\left|\nabla^{2}\left(\nabla_{z} \Phi(\mathbf{u})\right)\right|^{2} \mathrm{~d} x \mathrm{~d} t & \leq \frac{C}{(R-r)^{2}} \iint_{Q(x, t, R)}|\nabla \mathbf{u}|^{2} \mathrm{~d} x \mathrm{~d} t .
\end{aligned}
$$

Proof As before we will write $\mathbf{v}$ for $\nabla \Phi(\mathbf{u})$ and $f_{h}$ for Steklov average of $f$. Denote by $\xi \in C_{0}^{\infty}(\Omega)$ a smooth bump function supported in $\mathrm{B}_{R}(x) \subset \Omega$, which is identically one on $\mathrm{B}_{r}(x) \subset \Omega$ with $\|\nabla \xi\|_{\infty} \leq C /(R-r)$. Also denote by $\eta \in C_{0}^{\infty}\left(\left(t-R^{2}, t\right]\right)$ a function that is identically one on $\left[t-r^{2}, t\right]$ and supported in $\left[t-R^{2}, t\right]$ with $\left|\eta^{\prime}\right| \leq C /\left(R^{2}-r^{2}\right)$. Then multiplying equation

$$
\left(\mathbf{u}_{h}\right)_{t}-\Delta\left(\mathbf{v}_{h}\right)=0
$$

by $\left(\mathbf{v}_{h}\right)_{t} \xi^{2} \eta$ we obtain

$$
\int_{t-R^{2}}^{t} \int_{\Omega}\left(\mathbf{u}_{h}\right)_{t}\left(\mathbf{v}_{h}\right)_{t} \xi^{2} \eta+\nabla\left(\mathbf{v}_{h}\right) \nabla\left(\mathbf{v}_{h}\right)_{t} \xi^{2} \eta+\nabla\left(\mathbf{v}_{h}\right)\left(\mathbf{v}_{h}\right)_{t} 2 \xi \nabla \xi \eta \mathrm{d} x \mathrm{~d} t=0 .
$$

Using strict convexity on the first term, integrating the second term in time by parts and using Hölder inequality we obtain

$$
\begin{aligned}
& \int_{t-R^{2}}^{t} \int_{\Omega} \lambda\left|\left(\mathbf{u}_{h}\right)_{t}\right|^{2} \xi^{2} \eta \mathrm{d} x \mathrm{~d} t+\left.\int_{\Omega}\left|\nabla\left(\mathbf{v}_{h}\right)\right|^{2} \xi^{2} \eta\right|_{t} \mathrm{~d} x+\nabla\left(\mathbf{v}_{h}\right)\left(\mathbf{v}_{h}\right)_{t} 2 \xi \nabla \xi \eta \mathrm{d} x \mathrm{~d} t \\
& \quad \leq C\left\|\eta^{\prime}\right\|_{\infty} \int_{t-R^{2}}^{t} \int_{\Omega}\left|\nabla\left(\mathbf{v}_{h}\right)\right|^{2} \xi^{2} \mathrm{~d} x
\end{aligned}
$$


Finally using Hölder inequality on the third term and canceling part of the first term we compute

$$
\begin{gathered}
\int_{t-R^{2}}^{t} \int_{\Omega} \frac{\lambda}{2}\left|\left(\mathbf{u}_{h}\right)_{t}\right|^{2} \xi^{2} \eta \mathrm{d} x \mathrm{~d} t+\left.\int_{\Omega}\left|\nabla\left(\mathbf{v}_{h}\right)\right|^{2} \xi^{2} \eta\right|_{t} \mathrm{~d} x \\
\leq C\left(\left\|\eta^{\prime}\right\|_{\infty}+\|\nabla \xi\|_{\infty}^{2}\right) \int_{t-R^{2}}^{t} \int_{\Omega}\left|\nabla\left(\mathbf{v}_{h}\right)\right|^{2} \xi^{2} \mathrm{~d} x .
\end{gathered}
$$

Thus taking a limit as $h \rightarrow 0$ we deduce that $u_{t} \in L_{l o c}^{2}(\Omega)$ and derive estimate (15) as claimed. To prove (16), we use $\mathrm{H}^{2}$ estimates for the Laplacian to get the following for a fixed $t$ :

$$
\int_{\mathrm{B}_{r}(x) \times\{t\}}\left|\nabla^{2} \mathbf{v}\right|^{2} \mathrm{~d} x \leq \frac{C}{(R-r)^{2}} \int_{\mathrm{B}_{R}(x) \times\{t\}}|\nabla \mathbf{v}|^{2} \mathrm{~d} x+\int_{\mathrm{B}_{R}(x) \times\{t\}}\left|u_{t}\right|^{2} \mathrm{~d} x .
$$

Integrating in time and using estimate (15) we get

$$
\iint_{Q(x, t, r)}\left|\nabla^{2} \mathbf{v}\right|^{2} \mathrm{~d} x \mathrm{~d} t \leq \frac{C}{(R-r)^{2}} \iint_{Q(x, t, R)}|\nabla \mathbf{v}|^{2} \mathrm{~d} x \mathrm{~d} t .
$$

In addition to $\mathrm{H}^{2}$ estimates for weak solutions, bounded weak solutions of Eq. (8) are actually in $\mathrm{L}_{l o c}^{4}$. This is rather unusual since most quasi-linear equations do not have $\mathrm{L}^{4}$ estimates. The estimate is a formal consequence of $\mathrm{H}^{2}$ estimate and the assumption of boundness. Similar interpolation estimates have been used several times in the literature, see for instance paper by Choe [2].

Theorem 4 ( $\mathrm{L}^{4}$ estimate for bounded solutions) Let $\mathbf{u}$ be a weak bounded solution of the Eq. (8). Then $\nabla \mathbf{u}$ is locally in $\mathrm{L}^{4}$ and for $Q(x, t, r) \subsetneq Q(x, t, R) \subset \Omega_{T}$ we have the following estimate:

$$
\iint_{Q(x, t, r)}|\nabla \mathbf{u}|^{4} \mathrm{~d} x \mathrm{~d} t \leq \frac{C\|\mathbf{u}\|_{\infty}^{2}}{(R-r)^{2}} \iint_{Q(x, t, R)}|\nabla \mathbf{u}|^{2} \mathrm{~d} x \mathrm{~d} t .
$$

Proof Let us again denote $\nabla \Phi(\mathbf{u})$ by $\mathbf{v}$ and let $\tau: \mathbb{R} \rightarrow \mathbb{R}$ be a smooth increasing function that is linear on $(-\infty, 1]$ and constant on $[2, \infty)$. For some large enough constant $C_{1}, C_{1} \tau(z) \geq z\left(\tau^{\prime}(z)\right)^{2}$. Define $\tau_{\epsilon}$ as $\tau_{\epsilon}(x):=\tau(\epsilon x) / \epsilon$. Notice that $\left\|\tau_{\epsilon}^{\prime}\right\|_{\infty} \leq C_{0}$ and $C_{1} \tau_{\epsilon}(z) \geq z\left(\tau_{\epsilon}^{\prime}(z)\right)^{2}$ with constants independent of $\epsilon$. Letting $\xi$ be a smooth bump function as in the proof of Theorem above, multiply the Eq. (8) by $\mathbf{v} \tau_{\epsilon}\left(|\nabla \mathbf{v}|^{2}\right) \xi^{2}$ and integrate by parts to obtain

$$
\begin{aligned}
& \iint_{Q(x, t, R)} u_{t}^{i} v^{i} \tau_{\epsilon}\left(|\nabla \mathbf{v}|^{2}\right) \xi^{2}+|\nabla \mathbf{v}|^{2} \tau_{\epsilon}\left(|\nabla \mathbf{v}|^{2}\right) \xi^{2} \\
& \quad+2 v^{i} v_{x_{\alpha}}^{i} v_{x_{\beta}}^{j} \tau_{\epsilon}^{\prime}\left(|\nabla \mathbf{v}|^{2}\right) v_{x_{\alpha} x_{\beta}}^{j} \xi^{2}+2 v^{i} v_{x_{\alpha}}^{i} \tau_{\epsilon}\left(|\nabla \mathbf{v}|^{2}\right) \xi \xi_{x_{\alpha}} \mathrm{d} x \mathrm{~d} t=0 .
\end{aligned}
$$


Hence we compute

$$
\begin{aligned}
& \iint_{Q(x, t, R)}|\nabla \mathbf{v}|^{2} \tau_{\epsilon}\left(|\nabla \mathbf{v}|^{2}\right) \xi^{2} \mathrm{~d} x \mathrm{~d} t \leq \iint_{Q(x, t, R)} C_{2}\|\mathbf{v}\|_{\infty}^{2}\left|\mathbf{u}_{t}\right|^{2} \xi^{2}+\frac{1}{2} \tau_{\epsilon}^{2}\left(|\nabla \mathbf{v}|^{2}\right) \xi^{2} \\
& \quad+C_{3}\|\nabla \xi\|_{\infty}^{2}\|\mathbf{v}\|_{\infty}^{2}|\nabla \mathbf{v}|^{2}+C_{1}\|\mathbf{v}\|_{\infty}^{2}\left|\nabla^{2} \mathbf{v}\right|^{2} \xi^{2}+\frac{1}{4 C_{1}}|\nabla \mathbf{v}|^{4} \tau_{\epsilon}^{\prime}\left(|\nabla \mathbf{v}|^{2}\right)^{2} \xi^{2} \mathrm{~d} x \mathrm{~d} t .
\end{aligned}
$$

Simplifying and using estimates (15), (16) and $C_{1} \tau_{\epsilon}(z) \geq z\left(\tau_{\epsilon}^{\prime}(z)\right)^{2}$ we deduce

$$
\iint_{Q(x, t, r)}|\nabla \mathbf{v}|^{2} \tau_{\epsilon}\left(|\nabla \mathbf{v}|^{2}\right) \mathrm{d} x \mathrm{~d} t \leq \frac{C\|\mathbf{u}\|_{\infty}^{2}}{(R-r)^{2}} \iint_{Q(x, t, R)}|\nabla \mathbf{u}|^{2} \mathrm{~d} x \mathrm{~d} t .
$$

Taking a limit as $\epsilon \rightarrow 0$ we deduce by monotone convergence theorem that $\nabla \mathbf{u}$ is locally in $\mathrm{L}^{4}$ and the estimate as claimed in the statement of the Theorem.

Let us briefly review the concept of parabolic Hausdorff measure.

Definition 1 Let $\mathcal{H}^{k}: 2^{\mathbb{R}_{x}^{n} \times \mathbb{R}_{t}} \rightarrow \mathbb{R} \cup\{\infty\}$ be a set function defined as follows

$$
\mathcal{H}^{k}[X]:=\liminf _{\epsilon \rightarrow 0^{+}}\left\{\sum_{i} r^{k}\left(Q_{i}\right) \mid X \supseteq \bigcup Q_{i}, r\left(Q_{i}\right) \leq \epsilon\right\},
$$

where $r\left(Q_{i}\right)$ denotes the radius of the face of the ith cylinder. We call such a function a k-dimensional parabolic Hausdorff measure. The parabolic Hausdorff dimension of a set $X$ is defined as

$$
\operatorname{dim}_{p}(X):=\inf \left\{k \geq 0 \mid \mathcal{H}^{k}[X]=0\right\}
$$

It is a theorem to show that $\mathcal{H}^{k}$ is actually a measure. I refer you to the paper by Giaquinta and Giusti [9] for the discussion of parabolic Hausdorff measure, as well as the proof the following theorem:

Theorem 5 Let $f \in \mathrm{L}_{l o c}^{1}\left(\Omega_{T}\right)$, and for $0<k<n+2$, let

$$
S=\left\{(x, t) \in \Omega_{T}\left|\limsup _{r \rightarrow 0} r^{-k} \iint_{Q(x, t, r)}\right| f \mid \mathrm{d} x \mathrm{~d} t>0\right\} .
$$

Then we have $\mathcal{H}^{k}[S]=0$.

Remark 2 The fact that the gradient is actually locally in $\mathrm{L}^{4}$ implies that the singular set of a bounded solution has parabolic Hausdorff dimension smaller than $n-2$. As you may recall from Theorem 1 the singular set is contained in the set

$$
\left\{\left.(x, t) \in \Omega_{T}\left|\liminf _{R \rightarrow 0} \frac{1}{R^{n}} \iint_{Q(x, t, R)}\right| \nabla \mathbf{u}\right|^{2} \mathrm{~d} x \mathrm{~d} t>0\right\} .
$$


Now using the Hölder inequality and the fact that $\nabla \mathbf{u}$ is locally in $\mathrm{L}^{4}$ we compute

$$
\begin{aligned}
\frac{1}{R^{n}} \iint_{Q(x, t, R)}|\nabla \mathbf{u}|^{2} \mathrm{~d} x \mathrm{~d} t & \leq \frac{1}{R^{n}}\left(\iint_{Q(x, t, R)}|\nabla \mathbf{u}|^{4} \mathrm{~d} x \mathrm{~d} t\right)^{1 / 2}|Q(x, t, R)|^{1 / 2} \\
& \leq C R^{\frac{2-n}{2}}\left(\iiint_{Q(x, t, R)}|\nabla \mathbf{u}|^{4} \mathrm{~d} x \mathrm{~d} t\right)^{1 / 2} \\
& \leq C\left(R^{2-n} \iint_{Q(x, t, R)}|\nabla \mathbf{u}|^{4} \mathrm{~d} x \mathrm{~d} t\right)^{1 / 2}
\end{aligned}
$$

Hence the singular set must be contained in the set

$$
\left\{\left.(x, t) \in \Omega_{T}\left|\liminf _{R \rightarrow 0} \frac{1}{R^{n-2}} \iint_{Q(x, t, R)}\right| \nabla \mathbf{u}\right|^{4} \mathrm{~d} x \mathrm{~d} t>0\right\},
$$

which has $n-2$ parabolic Hausdorff measure zero.

\section{The key Lemma}

In this section I discuss the parabolic version of the lemma that seems to be key in the proof of everywhere regularity of some elliptic systems. Not surprisingly, it will turn out that the parabolic lemma is crucial to proving everywhere regularity of solutions to some types of parabolic systems. The elliptic lemma, to which I refer, is well known and the proof of it can be found in [8] in Chapter 7 as part of Theorem 1.1.

Before we proceed with the discussion of this elliptic lemma let us recall that coefficients $a_{\alpha \beta}$ are called strictly elliptic if there exists $\lambda>0$ such that

$$
a_{\alpha \beta} \xi^{\alpha} \xi^{\beta} \geq \lambda|\xi|^{2} \text { for all } \xi \in \mathbb{R}^{n} \text {. }
$$

Lemma 2 Suppose coefficients $a_{\alpha \beta}(x)$ are strictly elliptic, bounded and measurable. Let $u \in \mathrm{H}^{1}(\Omega), f \in \mathrm{L}^{1}(\Omega)$ be nonnegative functions satisfying

$$
-\left(a_{\alpha \beta} u_{x_{\beta}}\right)_{x_{\alpha}}+f \leq 0
$$

on $\Omega$. For any $x_{0} \in \Omega$ for which $B_{R_{0}}\left(x_{0}\right) \subset \Omega$ for some $R_{0}$, we have the following:

$$
\liminf _{R \rightarrow 0} \frac{1}{R^{n-2}} \int_{B_{R}\left(x_{0}\right)} f \mathrm{~d} x=0 .
$$

The proof of this lemma is rather simple and follows easily from the elliptic Harnack inequality. Because of the peculiar geometry of the parabolic Harnack inequality, the elliptic proof does not translate directly into the parabolic case. Instead, by adopting proof of elliptic lemma to parabolic equations, one is able to control $f$ on the cylinders whose top centers are slightly shifted back in time. In fact it is not true that $f$ can be controlled on the cylinders 
whose top centers are not fixed, without additional assumptions on $f$. It turns out, however, that the assumption that one needs to impose to prove the parabolic version of the lemma is satisfied in applications to everywhere regularity of parabolic systems. What we need to assume is that for some $\alpha>1$ the $\mathrm{L}^{\alpha}$ norm of $f$ on a cylinder is controlled by the $\mathrm{L}^{1}$ norm of $f$, perhaps on a larger cylinder.

Lemma 3 (Key Lemma) Suppose coefficients $a_{\alpha \beta}(x, t)$ are strictly elliptic, bounded and measurable. Let $u \in V\left(\Omega_{T}\right), f \in \mathrm{L}^{1}\left(\Omega_{T}\right)$ be nonnegative functions weakly satisfying

$$
u_{t}-\left(a_{\alpha \beta} u_{x_{\beta}}\right)_{x_{\alpha}}+f \leq 0
$$

on $\Omega_{T}$. Further suppose that for some $\alpha>1$ our $f$ satisfies the following:

$$
\left(\iint_{Q_{R}} f^{\alpha} \mathrm{d} x \mathrm{~d} t\right)^{1 / \alpha} \leq C \iint_{Q_{4 R}} f \mathrm{~d} x \mathrm{~d} t,
$$

for all $Q_{R} \subset Q_{4 R} \subset \Omega_{T}$. Then for any $\left(x_{0}, t_{0}\right) \in \Omega_{T}$ for which

$$
B_{R_{0}}\left(x_{0}\right) \times\left(t_{0}-R_{0}^{2}, t_{0}+R_{0}^{2}\right) \subset \Omega_{T}, \text { for some } R_{0},
$$

we have the following:

$$
\liminf _{R \rightarrow 0} \frac{1}{R^{n}} \iint_{Q\left(x_{0}, t_{0}, R\right)} f \mathrm{~d} x \mathrm{~d} t=0 .
$$

Proof First of all notice that $u$ is a priori locally bounded due to supremum estimates for subsolutions of parabolic equations (see Theorem 6.17 in [11]). Fix $0<\sigma \leq 1 / 4$. Set

$$
R_{i}:=\sigma^{i} R_{0}, \quad Q_{i}:=Q\left(x_{0}, t_{0}, R_{i}\right), \quad M_{i}:=\sup _{Q_{i}} u
$$

and

$$
\begin{aligned}
& Q_{i}^{\prime}:=Q\left(x_{0}, t_{0}-4 \sigma^{2} R_{i}^{2},\left(1-8 \sigma^{2}\right)^{1 / 2} R_{i}\right), \\
& Q_{i}^{\prime \prime}:=Q\left(x_{0}, t_{0}-2 \sigma^{2} R_{i}^{2},\left(1-4 \sigma^{2}\right)^{1 / 2} R_{i}\right) .
\end{aligned}
$$

We divide the proof in three steps. In the first step we show that for any $\sigma \in(0,1 / 4]$ we have

$$
\lim _{i \rightarrow \infty} \frac{1}{R_{i}^{n}} \iint_{Q_{i}^{\prime}} f \mathrm{~d} x \mathrm{~d} t=0 .
$$

Once we have done that, we show that we can control $f$ on $Q_{i} \backslash Q_{i}^{\prime}$ with the help of the assumption on $f$. Finally we will put it all together to conclude the lemma.

Step 1. Fix $i$, and set $z:=M_{i}-u$. We see that $z \geq 0$ on $Q_{i}$ and $z$ satisfies

$$
z_{t}-\left(a_{\alpha \beta} z_{x_{\beta}}\right)_{x_{\alpha}} \geq f
$$

In particular, due to parabolic Harnack inequality (see Theorem 6.24 in [11])

$$
\iint_{Q_{i}^{\prime \prime}} z \mathrm{~d} x \mathrm{~d} t \leq C \inf _{Q_{i+1}} z .
$$


Let $w$ solve backward time parabolic equation

$$
-w_{t}-\left(a_{\alpha \beta} w_{x_{\alpha}}\right)_{x_{\beta}}=\frac{1}{R_{i}^{2}} \chi_{Q_{i}^{\prime \prime}}
$$

on $Q_{i}$ with $w \equiv 0$ on the backward time parabolic boundary, that is

$$
w \equiv 0 \text { on }\left(\partial \mathrm{B}_{R_{i}}\left(x_{0}\right) \times\left[t_{0}-R_{i}^{2}, t_{0}\right]\right) \cup\left(B_{R_{i}}\left(x_{0}\right) \times\left\{t_{0}\right\}\right) .
$$

At this point if $z w$ were actually differentiable in time, we would multiply Eq. (25) by $z w$ and integrate by parts to obtain

$$
\iint_{Q_{i}}-z\left(\frac{w^{2}}{2}\right)_{t}+a_{\alpha \beta} z_{\beta}\left(\frac{w^{2}}{2}\right)_{x_{\alpha}}+a_{\alpha \beta} w_{x_{\alpha}} w_{x_{\beta}} z \mathrm{~d} x \mathrm{~d} t=\frac{1}{R_{i}^{2}} \iint_{Q_{i}^{\prime \prime}} z w \mathrm{~d} x \mathrm{~d} t,
$$

and since $z$ satisfies Eq. (23) we would conclude that

$$
\frac{1}{R_{i}^{n}} \iint_{Q_{i}^{\prime}} f\left(\frac{w^{2}}{2}\right) \mathrm{d} x \mathrm{~d} t \leq \frac{1}{R_{i}^{n+2}} \iint_{Q_{i}^{\prime \prime}} z w \mathrm{~d} x \mathrm{~d} t .
$$

In general we cannot expect $z w$ to be differentiable in time. To obtain Eq. (26) rigorously one would need to use Steklov average

$$
(z w)^{h}(x, t):=\frac{1}{h} \int_{t-h}^{t} z(x, \tau) w(x, \tau) \mathrm{d} \tau
$$

as a test function in (25). However, we will not do this here, instead I refer the reader to Lemma 6.1 in [11], where similar computation has been carried out.

Now, since $w$ solves (25), by strong maximal principle $w \geq \theta>0$ on $Q_{i}^{\prime}$ and also $w \leq C$ on $Q_{i}$, with bounds independent of $i$ (one can see this by scaling for example). Therefore, combining this observation with inequality (24), we obtain

$$
\frac{1}{R_{i}^{n}} \iint_{Q_{i}^{\prime}} f \mathrm{~d} x \mathrm{~d} t \leq C-\iint_{Q_{i}^{\prime \prime}} z \mathrm{~d} x \mathrm{~d} t \leq C \inf _{Q_{i+1}} z=C\left(M_{i}-M_{i+1}\right) .
$$

However, since

$$
\sum_{i=0}^{\infty} M_{i}-M_{i+1} \leq \sup _{Q_{0}} u
$$

we conclude that

$$
\frac{1}{R_{i}^{n}} \iint_{Q_{i}^{\prime}} f \mathrm{~d} x \mathrm{~d} t \rightarrow 0 \quad \text { as } \quad i \rightarrow \infty .
$$

Step 2. Let $A$ be some measurable set. We will show that for all $Q_{R} \subset Q_{8 R} \subset Q_{R_{0}}$ and for all $\epsilon$, there exists $\delta$ such that if $\left|A \cap Q_{R}\right| \leq \delta\left|Q_{R}\right|$, then

$$
\frac{1}{R^{n}} \iint_{A \cap Q_{R}} f \mathrm{~d} x \mathrm{~d} t \leq \epsilon .
$$


First, we can easily deduce by the argument similar to the one in part 1 , that

$$
\frac{1}{(4 R)^{n}} \iint_{Q_{4 R}} f \mathrm{~d} x \mathrm{~d} t \leq C,
$$

where constant is independent of $R$.

We now use (21) to conclude that

$$
\begin{aligned}
\iint_{A \cap Q_{R}} f \mathrm{~d} x \mathrm{~d} t & \leq\left(\frac{1}{\left|Q_{R}\right|} \iint_{A \cap Q_{R}} f^{\alpha} \mathrm{d} x \mathrm{~d} t\right)^{1 / \alpha}\left|Q_{R}\right|^{1 / \alpha}\left|A \cap Q_{R}\right|^{1-1 / \alpha} \\
& =C\left|Q_{R}\right|^{1 / \alpha}\left|A \cap Q_{R}\right|^{1-1 / \alpha} \int f f \mathrm{~d} x \mathrm{~d} t \\
& =C\left(\frac{\left|A \cap Q_{R}\right|}{\left|Q_{R}\right|}\right)^{1-1 / \alpha} \iint_{Q_{4 R}} f \mathrm{~d} x \mathrm{~d} t \leq C_{1} R^{n} \delta^{1-1 / \alpha} .
\end{aligned}
$$

Above, the last inequality follows by (27).

Step 3. Finally we put everything together. Fix $\epsilon>0$. First notice that by choosing $\sigma$ small enough we can make

$$
\left|Q_{i} \backslash Q_{i}^{\prime}\right| \leq \delta\left|Q_{i}\right|,
$$

where $C_{1} \delta^{1-1 / \alpha}<\epsilon / 2$. Then by first step we can find $i>2$ such that

$$
\frac{1}{R_{i}^{n}} \iint_{Q_{i}^{\prime}} f \mathrm{~d} x \mathrm{~d} t \leq \epsilon / 2 .
$$

Finally, the above together with conclusion of second step gives us

$$
\frac{1}{R_{i}^{n}} \iint_{Q_{i}} f \mathrm{~d} x \mathrm{~d} t=\frac{1}{R_{i}^{n}} \iint_{Q_{i}^{\prime}} f \mathrm{~d} x \mathrm{~d} t+\frac{1}{R_{i}^{n}} \iint_{Q_{i} \backslash Q_{i}^{\prime}} f \mathrm{~d} x \mathrm{~d} t<\epsilon .
$$

Now we are in position to apply our key Lemma to deduce crucial importance of entropy in questions of everywhere regularity for parabolic systems.

Theorem 6 (Entropy condition) Let $\mathbf{u}$ be weak solution of (3). Suppose there exists $\phi \in$ $V\left(\Omega_{T}\right)$ that together with $\mathbf{u}$ weakly satisfy the following inequality:

$$
\phi_{t}-\left(a_{\alpha \beta} \phi_{x_{\beta}}\right)_{x_{\alpha}}+\lambda|\nabla \mathbf{u}|^{2} \leq 0,
$$

where $a_{\alpha \beta}$ are bounded and strictly elliptic. Then $\mathbf{u}$ is everywhere Hölder continuous on the interior of $\Omega_{T}$.

Proof Since $\mathbf{u}$ satisfies condition (6), we see immediately that conditions of the key Lemma 3 are satisfied. Therefore, we conclude that

$$
\liminf _{R \rightarrow 0} \frac{1}{R^{n}} \iint_{Q\left(X_{0}, R\right)}|\nabla \mathbf{u}|^{2} \mathrm{~d} x \mathrm{~d} t=0 .
$$

However, this is precisely the condition (5) of Theorem 1 . Hence we conclude that $\mathbf{u}$ is everywhere Hölder continuous on the interior of $\Omega_{T}$. 


\section{Everywhere regularity of certain diffusion systems}

In this section we come back to the discussion of weak solutions of the diffusion system (8), imposing additional requirement that $\Phi(z)$ is a function of only the norm of $z$. As we will see this allows us to conclude much more about solutions of this equation. In particular solutions are bounded if they are bounded initially and at the boundary. More importantly, solutions are actually everywhere Hölder continuous and thus smooth if $\Phi$ is.

First I will show that in the case when $\Phi$ is a functions of only the norm, weak solutions of Eq. (8) that are bounded initially and bounded at the boundary will remain bounded for all time.

Theorem 7 (Boundedness) Let $\mathbf{u}$ be a weak solution of the generalized diffusion Eq. (8). Also suppose that $\|\mathbf{u}(\cdot, 0)\|_{L^{\infty}(\Omega)}<\infty$ and $\|\mathbf{u}(\cdot, t)\|_{L^{\infty}(\partial \Omega)}<\infty$ for all $t \in[0, T]$. Then $\|\mathbf{u}\|_{L^{\infty}(\Omega)}<\infty$ and we have

$$
\|\mathbf{u}(\cdot, t)\|_{\mathrm{L}^{\infty}(\Omega)} \leq \max \left\{\|\mathbf{u}(\cdot, 0)\|_{\mathrm{L}^{\infty}(\Omega)}, \sup _{s \in[0, t]}\|\mathbf{u}(\cdot, s)\|_{\mathrm{L}^{\infty}(\partial \Omega)}\right\} .
$$

Proof Set $B$ to

$$
B=\max \left\{\|\mathbf{u}(\cdot, 0)\|_{\mathrm{L}^{\infty}(\Omega)}, \sup _{s \in[0, t]}\|\mathbf{u}(\cdot, s)\|_{\mathrm{L}^{\infty}(\partial \Omega)}\right\} .
$$

Fix $\epsilon>0$ that is less than one. Let $\gamma: \mathbb{R}_{+} \rightarrow \mathbb{R}$ be a smooth convex function which is identically zero on $[0, B+\epsilon]$, positive and increasing otherwise, and linear on $[B+1, \infty)$. Also set $\Gamma(z)$ to $\gamma(|z|)$. Then we compute

$$
\begin{aligned}
\frac{\mathrm{d}}{\mathrm{d} t} \int_{\Omega} \Gamma(\mathbf{u}(x, t)) \mathrm{d} x & =\int_{\Omega} \Gamma_{z_{i}} u_{t}^{i} \mathrm{~d} x \\
& =\int_{\Omega}\left(\Gamma_{z_{i}} \Phi_{z_{i} z_{j}} u_{x_{\alpha}}^{j}\right)_{x_{\alpha}}-\Gamma_{z_{i} z_{k}} \Phi_{z_{k} z_{j}} u_{x_{\alpha}}^{i} u_{x_{\alpha}}^{j} \mathrm{~d} x \\
& \leq \int_{\partial \Omega} \Gamma_{z_{i}} \Phi_{z_{i} z_{j}} u_{x_{\alpha}}^{j} v_{\alpha} \mathrm{d} S \\
& =0 .
\end{aligned}
$$

The inequality is true because Hessians of two functions of only the norm commute and both $\Gamma$ and $\Phi$ are convex. The last equality is true because $\gamma$ is identically zero on $[0, B+\epsilon]$ and $|\mathbf{u}|$ is less than or equal to $B$ on the boundary. Since $\Gamma(\mathbf{u})$ is positive and initially zero we conclude that $\Gamma$ is zero up to time $t$ and thus $\|\mathbf{u}(\cdot, t)\|_{\infty} \leq B+\epsilon$. Since the inequality is true for all $\epsilon>0$ the Theorem follows.

The next lemma will show that if we suppose that $\Phi(z)$ is a function only of the norm of $z$, then there exists an entropy that satisfies conditions of Theorem 6.

Lemma 4 Let $\mathbf{u}$ be a weak bounded solution of (8) and suppose $\Phi$ is of the form

$$
\Phi(\mathbf{u})=\phi(|\mathbf{u}|) .
$$

Then there is a continuously differentiable, strictly increasing function $\gamma: \mathbb{R} \rightarrow \mathbb{R}$ such that $\phi=\phi(|\mathbf{u}|)$ weakly satisfies the following inequality: 


$$
\phi_{t}-\Delta(\gamma(\phi))+\lambda^{2}|\nabla \mathbf{u}|^{2} \leq 0 \text {. }
$$

Proof First of all, without loss of generality we can suppose that $\Phi(0)=0$. Notice that $\phi$ satisfies the following equation weakly:

$$
\phi_{t}-\left(\Phi_{z_{i}} \Phi_{z_{i} z_{j}} u_{x_{\alpha}}^{j}\right)_{x_{\alpha}}+\left|\nabla_{x}(\nabla \Phi(\mathbf{u}))\right|^{2}=0 .
$$

Looking at the quantity inside the divergence term we see that it is equal to

$$
\Phi_{z_{i}} \Phi_{z_{i} z_{j}} u_{x_{\alpha}}^{j}=\left(\frac{1}{2}|\nabla \Phi(\mathbf{u})|^{2}\right)_{x_{\alpha}} .
$$

Since $\Phi(z)=\phi(|z|)$, we observe that

$$
\frac{1}{2}|\nabla \Phi(\mathbf{u})|^{2}=\frac{1}{2} \phi^{\prime}(|\mathbf{u}|)^{2} .
$$

Let $\psi$ be the continuous inverse of $\phi$. Set

$$
\gamma(z)=\int_{0}^{z} \phi^{\prime \prime}(\psi(t)) \mathrm{d} t .
$$

Then multiplying $\gamma^{\prime}(\phi(z))$ by $\phi^{\prime}(z)$ and integrating, we see that $\gamma$ and $\phi$ satisfy

$$
\gamma(\phi(z))=\frac{1}{2} \phi^{\prime}(z)^{2} .
$$

This $\gamma$ is continuously differentiable and strictly increasing, since $\phi$ is strictly convex. Therefore we conclude that $\phi$ satisfies

$$
\phi_{t}-\Delta(\gamma(\phi))+\left|\nabla_{x}(\nabla \Phi(\mathbf{u}))\right|^{2}=0,
$$

and due to strict convexity the last term on the left hand side is greater or equal to $\lambda^{2}|\nabla \mathbf{u}|^{2}$.

Now we are in position to use the above Lemma 4 together with Theorem 6 to deduce

Theorem 8 Weak solutions of Eq. 8 are Hölder continuous in $\Omega_{T}$.

Proof Lemma 4 tells us, that for $\mathbf{u}$ a weak bounded solution of (8), there exists $\phi$ satisfying

$$
\phi_{t}-\left(a \phi_{x_{\alpha}}\right)_{x_{\alpha}}+\lambda^{2}|\nabla \mathbf{u}|^{2} \leq 0,
$$

where $a(x, t):=\gamma^{\prime}(\phi(x, t))$. However, this is precisely the condition of Theorem 6 . Therefore, we conclude the proof.

\section{Strongly coupled parabolic systems}

One of the earliest nontrivial examples of quasi-linear parabolic systems whose solutions have interior everywhere regularity was due to Wiegner [13]. These are the so-called strongly coupled parabolic systems of the following form:

$$
u_{t}^{i}-\left(a_{\alpha \beta} u_{x_{\beta}}^{i}+c_{\alpha \beta}^{i} H_{x_{\beta}}\right)_{x_{\alpha}}=0,
$$


where

1. $H=H(\mathbf{u})$ is a function of $\mathbf{u}$;

2. $A_{\alpha \beta}^{i j}:=a_{\alpha \beta} \delta^{i j}+c_{\alpha \beta}^{i} H_{z_{j}}$ and $a_{\alpha \beta}$ are strictly elliptic in the sense that

$$
\lambda|\xi|^{2} \leq A_{\alpha \beta}^{i j} \xi_{\alpha}^{i} \xi_{\beta}^{j} \text {, and } \lambda|\zeta|^{2} \leq a_{\alpha \beta} \zeta^{i} \zeta^{j}, \text { for all } \xi \in \mathbb{R}^{n N}, \zeta \in \mathbb{R}^{n}
$$

3. $H(z)$ is twice continuously differentiable and $\lambda|\zeta|^{2} \leq H_{z_{i} z_{j}} \zeta^{i} \zeta^{j}$;

4. $a_{\alpha \beta}$ and $c_{\alpha \beta}^{i}$ are bounded.

After Wiegner, Dung [6] also worked on these types of system. However, neither Wiegner's nor Dung's proofs of everywhere regularity for solutions of strongly coupled parabolic systems reduce to something analogous to the key Lemma 3. It is instructive to prove everywhere regularity of weak solutions to (30) using the key Lemma to illustrate an underlying similarity between strongly coupled parabolic systems and generalized diffusion Eqs. (8). It appears that for both systems discussed in this paper the existence of an entropy is crucial for everywhere regularity of their solutions.

Remark 3 When $\Phi$ only depends on the norm of the gradient, diffusion system (8) actually has the form of a strongly coupled system, except with possibly non-convex $H$. Indeed, if $\Phi(z)=\phi(|z|)$, then

$$
\Phi_{z_{i} z_{j}}(z)=\frac{\phi^{\prime}(|z|)}{|z|} \delta_{i j}+\frac{z_{i}}{|z|}\left(\phi^{\prime \prime}(|z|)-\frac{\phi^{\prime}(|z|)}{|z|}\right) \frac{z_{j}}{|z|},
$$

therefore, with

$$
a_{\alpha \beta}=\frac{\phi^{\prime}(|\mathbf{u}|)}{|\mathbf{u}|} \delta_{\alpha \beta}, \quad c_{\alpha \beta}^{i}=\frac{u^{i}}{|\mathbf{u}|} \delta_{\alpha \beta},
$$

and

$$
H(z)=\phi^{\prime}(|z|)-\int_{0}^{|z|} \frac{\phi^{\prime}(s)}{s} \mathrm{~d} s,
$$

system (8) has the form (30).

We can prove an interior everywhere regularity result for strongly coupled parabolic systems rather easily using Theorem 6. As in the previous section I will show existence of an entropy.

Lemma 5 Let $\mathbf{u} \in V\left(\Omega_{T} ; \mathbb{R}^{N}\right)$ be a weak bounded solution of (30), then for some large enough s there is a positive constant c such that $v:=e^{s H}$ is a subsolution of the following equation:

$$
v_{t}-\left(A_{\alpha \beta} v_{x_{\beta}}\right)_{x_{\alpha}}+c|\nabla \mathbf{u}|^{2} \leq 0 .
$$

Proof We compute

$$
\begin{aligned}
\left(e^{s H}\right)_{t} & =s e^{s H} H_{z_{i}}\left(a_{\alpha \beta} u_{x_{\beta}}^{i}+c_{\alpha \beta}^{i} H_{x_{\beta}}\right)_{x_{\alpha}} \\
& =\left(A_{\alpha \beta}\left(e^{s H}\right)_{x_{\beta}}\right)_{x_{\alpha}}-\left(s e^{s H} H_{z_{i}}\right)_{x_{\alpha}}\left(a_{\alpha \beta} u_{x_{\beta}}^{i}+c_{\alpha \beta}^{i} H_{x_{\beta}}\right) .
\end{aligned}
$$


The last term on the right becomes

$$
\begin{aligned}
& \left(s e^{s H} H_{z_{i}}\right)_{x_{\alpha}}\left(a_{\alpha \beta} u_{x_{\beta}}^{i}+c_{\alpha \beta}^{i} H_{x_{\beta}}\right)=s^{2} e^{s H} A_{\alpha \beta} H_{x_{\alpha}} H_{x_{\beta}} \\
& \quad+s e^{s H} H_{z_{i} z_{j}} a_{\alpha \beta} u_{x_{\alpha}}^{i} u_{x_{\beta}}^{j}+s e^{s H} H_{z_{i} z_{j}} c_{\alpha \beta}^{i} H_{x_{\beta}} u_{x_{\alpha}}^{i} \\
& \geq s e^{s H}\left(\lambda s|\nabla H|^{2}+\lambda|\nabla \mathbf{u}|^{2}-C(\epsilon)|\nabla H|^{2}-\epsilon|\nabla \mathbf{u}|^{2}\right) \geq \frac{\lambda}{2} s e^{s H}|\nabla \mathbf{u}|^{2} .
\end{aligned}
$$

The last inequality follows by first making $\epsilon$ small and then $s$ large. Since $\mathbf{u}$ is bounded, we have $H$ is bounded from below. Therefore, for some $c, v$ satisfies Eq. (31) as claimed.

At this point we immediately conclude that conditions of Theorem 6 are satisfied. Therefore we have established.

Theorem 9 Bounded weak solutions of (30) are Hölder continuous in $\Omega_{T}$.

Acknowledgements The author would like to thank his thesis advisor L. C. Evans for his continuous support and helpful advise.

Open Access This article is distributed under the terms of the Creative Commons Attribution Noncommercial License which permits any noncommercial use, distribution, and reproduction in any medium, provided the original author(s) and source are credited.

\section{References}

1. Bae, H.-O., Choe, H.J.: Regularity for certain nonlinear parabolic systems. Commun. Partial Differ. Equ. 29(5-6), 611-645 (2004)

2. Choe, H.J.: Interior behaviour of minimizers for certain functionals with nonstandard growth. Nonlinear Anal. 19(10), 933-945 (1992)

3. DiBenedetto, E.: Degenerate Parabolic Equations. Universitext. Springer-Verlag, USA (1993)

4. DiBenedetto, E., Friedman, A.: Regularity of solutions of nonlinear degenerate parabolic systems. J. Reine Angew. Math. 349, 83-128 (1984)

5. DiBenedetto, E., Friedman, A.: Hölder estimates for nonlinear degenerate parabolic systems. J. Reine Angew. Math. 357, 1-22 (1985)

6. Dung, L.: Hölder regularity for certain strongly coupled parabolic systems. J. Differ. Equ. 151(2), 313-344 (1999)

7. Duzaar, F., Mingione, G.: Second order parabolic systems, optimal regularity, and singular sets of solutions. Ann. Inst. H. Poincare Anal. Non Line aire 22(6), 705-751 (2005)

8. Giaquinta, M.: Multiple Integrals in the Calculus of Variations and Nonlinear Elliptic Systems. Princeton University Press, Princeton (1983)

9. Giaquinta, M., Giusti, E.: Partial regularity for the solutions to nonlinear parabolic systems. Ann. Mat. Pura Appl. 4(97), 253-266 (1973)

10. Giaquinta, M., Struwe, M.: On the partial regularity of weak solutions of nonlinear parabolic systems. Math. Z. 179(4), 437-451 (1982)

11. Lieberman, G.M.: Second Order Parabolic Differential Equations. World Scientific Publishing Co. Inc., Singapore (1996)

12. Uhlenbeck, K.: Regularity for a class of non-linear elliptic systems. Acta Math. 138(3-4), 219-240 (1977)

13. Wiegner, M.: Global solutions to a class of strongly coupled parabolic systems. Math. Ann. 292(4), 711-727 (1992) 